PANORAMA ECONÓMICO, Volumen VI, No. 11, julio-diciembre, 2010, pp. 115-138

\title{
FORMACIÓN DE CAPITAL HUMANO Y
}

\section{AHORRO INTERGENERACIONAL}

\author{
Alí AAlí Bujari* \\ Humberto Ríos Bolivar** \\ (Recibido: Agosto, 2010 / Aprobado: Octubre, 2010)
}

\begin{abstract}
RESUMEN
La acumulación de capital, y por lo tanto el ahorro, tienen un papel fundamental en el crecimiento económico. El objetivo de este artículo es modelar las principales situaciones en que, para un agente racional resulta óptimo no ahorrar sino dedicar sus recursos a otros objetivos, como puede ser la acumulación de capital humano. Desde el punto de vista de su estructura económica, la inversión en capital humano sucede en dos etapas: la primera corresponde a la niñez y la segunda a la etapa laboral de la vida. Para analizar el problema de las necesidades infantiles, agregamos un período de vida más al modelo de Diamond (1965), la etapa infan-
\end{abstract}

* Pasante del programa de Doctorado en Ciencias Económicas, Sección de Estudios de Posgrado e Investigación, ESE-IPN. Correo electrónico:<alibujari@yahoo.es>.

** Profesor de la Sección de Estudios de Posgrado e Investigación, ESE-IPN. Correo electrónico: $<$ hrios@ipn.mx>. 
til. Examinamos el comportamiento diferenciado de los agentes según su riqueza cuando, al decidir sobre la educación de sus hijos deciden sobre la formación de capital humano. Encontramos que una familia representativa de pocos recursos dedica su ingreso a la formación de capital humano y por lo tanto no ahorra. Esto sucede aún más en una economía abierta en que la tasa de interés del exterior es más baja que la doméstica.

Palabras clave: Capital humano, ahorro, crecimiento económico, etapa infantil Clasificación JEL : D24, O13, O47, Q10

\begin{abstract}
The accumulation of capital, and therefore savings, have a key role in economic growth. The aim of this paper is to model the main situations where, for a rational agent is optimal not to save but to devote its resources to other objectives, such as human capital accumulation. From the point of view of its economic structure, investment in human capital takes place in two stages: the first is the childhood and the second labor stage of life. To analyze the problem of children's needs, we added a further life span model of Diamond (1965), the infant stage. Examine the differential behavior of agents based on their wealth when, in deciding on the education of their children decide on human capital formation. We found that a representative family of few resources spends his income to the formation of human capital and therefore not saved. This happens even in an open economy where the foreign interest rate is lower than domestic

Keywords: Human capital, savings, economic growth, infancy

JEL Classification: D24, O13, O47, Q10
\end{abstract}

\title{
1. INTRODUCCIÓN
}

La acumulación de capital, y por lo tanto el ahorro, tienen un papel fundamental en el crecimiento económico. El objetivo fundamental de los modelos de crecimien- 
to, endógeno en modelos continuos o discretos de optimización intertemporal, es explicar la dinámica de la acumulación. Sin embargo, especialmente cuando se estudian estos modelos desde el punto de vista de los países menos desarrollados, uno de sus puntos débiles resulta ser que no explican la falta de ahorro. Este es un problema que en buena medida surge del modelo neoclásico de maximización de utilidad, en el que el agente siempre encontrará algún nivel óptimo de ahorro. También surge de la falta de atención a los problemas de distribución pues, solamente es posible que una parte de la población no ahorre si otra está ahorrando. Esta postura teórica contrasta con la de los clásicos, para quienes la pobreza impedía el ahorro cuya fuente principal eran los beneficios de la inversión. El objetivo es modelar las principales situaciones en que resulta óptimo para un agente racional no ahorrar, sino dedicar sus recursos a otros objetivos.

Intuitivamente, para una familia de pocos recursos, satisfacer las necesidades de los hijos puede tener tal prioridad que le impida ahorrar. En la etapa infantil existen necesidades que cumplen un papel formativo, algunas de estas necesidades tienen la naturaleza de consumo y podrían implicar que estas preferencias no son separables a lo largo de las distintas etapas, contribuyendo el consumo en la infancia al bienestar durante toda la vida. Sin embargo esto no alteraría de forma cualitativa los modelos que explican el ahorro, a menos que se cambie la concepción de la forma en que el consumo brinda utilidad, punto de vista explorado en un artículo paralelo elaborado por Mayer (1997). Este artículo se centra en aquellos aspectos de la formación en la etapa de vida anterior al trabajo que tienen repercusiones sobre el ingreso del futuro adulto y que, por lo tanto, toman la forma de capital humano productivo.

En los modelos de capital humano de Usawa (1965), Lucas (1988), Becker et al. (1990), Rebelo (1991), se considera, por una parte, que el stock de capital humano se transmite de una generación a otra y, por otra, que existe un proceso de producción del capital humano. Examinamos el comportamiento diferenciado de los agentes según su riqueza, cuando al decidir sobre la educación de sus hijos deciden sobre la producción de capital humano. Las familias tienen a su disposición una variedad de maneras de dedicar recursos a la educación de sus hijos con el objeto de mejorar sus posibilidades de ingreso futuro. En general podemos considerar que, 
estas posibilidades que constituyen la función de inversión en capital humano tienen rendimientos decrecientes en los recursos invertidos y que, por lo tanto, la familia invertirá en ellos hasta que el rendimiento iguale el de la tasa de interés. Asimismo, antes de llegar a ese punto no ahorrará, si pensamos a largo plazo e ignoramos otros motivos del ahorro como son el precaucional. Si la familia cuenta con pocos recursos, posiblemente se iguale su tasa de substitución intemporal con el rendimiento de la inversión en capital humano a un nivel mayor que la tasa de interés, que se presenta como un precio que tiene que tomar independientemente de sus acciones, y por lo tanto no participe en el mercado de ahorro.

Implícitamente este planteamiento involucra una restricción de crédito, pues las familias preferirían pedir prestado para financiar una mayor inversión en capital humano ya que ésta tiene un mayor rendimiento para ellas. Una de las consecuencias es que la inversión en capital humano presenta así una función redistributiva, si consideramos que el ingreso del sector pobre de la población, en tanto no ahorra debido a estas razones, tiene una mayor tasa de crecimiento que el del sector que sí ahorra. En el artículo paralelo arriba mencionado la situación es al revés, y se presenta una situación en que el ingreso de las familias que no ahorran no crece.

La inversión en capital humano de las familias para los hijos se introduce de manera natural en los modelos de generaciones traslapadas. Modigliani (1986) enfatiza el papel de las restricciones de crédito en aspectos económicos diferentes a la acumulación de capital físico. Al estudiar la restricción de crédito, Jappelli y Pagano (1994) concluyen que incrementa el crecimiento. De Gregorio (1996), en un estudio semejante al nuestro pero que no estudia la distribución, concluye que los efectos sobre el crecimiento pueden ser de ambos signos; además, menciona un trabajo de Christou (1993) con resultados similares a los de él y a los de Buiter y Kletzer (1992), en que la restricción de crédito disminuye la acumulación de capital. Barro, Mankiw y Sala-I-Martin (1995) discuten el efecto de esta restricción sobre la convergencia. Desde el punto de vista de su estructura económica, la inversión en capital humano sucede en dos etapas. La primera corresponde a la niñez y no sustituye el trabajo. La segunda corresponde a la etapa laboral de la vida $y$, para realizar esta inversión el agente debe dejar de trabajar. Nos concentramos en la primera de éstas que parece muy urgente en los países en vías de desarrollo. 
Hay que mencionar que, los estudios arriba mencionados se ocupan de la segunda y muchos de sus resultados dependen de dicha disyuntiva.

Para analizar el problema de las necesidades infantiles agregamos un período de vida más al modelo de Diamond (1965), correspondiente a la etapa infantil. En esta etapa no hay ingresos sino solamente hay consumo provisto por los adultos a sus hijos e inversión en capital humano. El altruismo es un componente esencial de nuestro punto de vista pues, sin éste no habría el cuidado de los hijos ni la posibilidad de que los adultos hagan transferencias hacia los jóvenes aún al grado de quedar en una situación de dependencia en la vejez. Encontramos que pensar en la familia como un agente que en cada tiempo maximiza simultáneamente la utilidad de los niños, jóvenes y viejos que la constituyen es equivalente, en el caso de utilidad aditiva, a pensar en cada individuo como maximizador de su propia utilidad y de la de generaciones siguientes y anteriores, como lo señalan Abel $(1985,1987)$ y Kimball (1987). Es entonces que presentamos este trabajo para exponer la alternativa, la cual tiene las siguientes ventajas. Primero, es más atractivo como concepto sociológico. Aunque no discutimos cómo se formaría la decisión agregada de la familia, el modelo de decisión individual por parte de los adultos, en que simultáneamente deciden su consumo como viejos, no es particularmente satisfactorio, y queda abierta esta representación a otras explicaciones. Segundo, el modelo solamente considera consumos presentes y futuros, en lugar de también pasados. Tercero, las decisiones de consumo de cada tiempo son independientes. Esto implica que, por ejemplo, puedan retomarse en el caso de shocks de productividad positivos o negativos modificando consistentemente el consumo de los viejos.

Es fundamental que la ausencia de ahorro esté intrínsecamente ligada con la desigualdad en la distribución de la riqueza. Para entender esto basta pensar que, si los agentes son idénticos la tasa de interés tiene que subir al nivel al que incentive el ahorro pues de lo contrario no habría capital. Por ello, manteniendo la hipótesis de individuos idénticos, dividimos las familias en dos poblaciones uniformes, cada una con niveles de riqueza diferentes, y estudiamos la economía agregada.

Así, trabajaremos con un modelo de tres generaciones traslapadas y dos sectores de población. Con el objeto de simplificarlo nuestra función de producción 
tiene como insumos solamente capitales físico y humano, mas no el trabajo. La función de producción es homogénea de grado uno debido al proceso competitivo en el que se da la producción. Consideramos que esta función agregada de producción incluye la producción del servicio educativo pero, quien lo adquiere percibe rendimientos decrecientes del servicio en su producción de capital humano efectivo remunerable. Si bien esto es una simplificación, no es irrealista pensar que la esencia de la producción de capital humano no radica en la proporción de sus insumos, sino en que el producto es remunerado a través de su utilización en el trabajo; esto nos evita adicionar al modelo las complicaciones de un segundo sector.

La función de inversión en capital humano es de rendimientos decrecientes por lo cual, sin considerar externalidades que en este caso incluirían la herencia social de capital humano, no se genera crecimiento. Consideraremos dos casos. En el primero el capital humano promedio de la sociedad aumenta la productividad del servicio educativo hasta convertirse en un motor de crecimiento. En el segundo no aumenta tanto la productividad, pero el crecimiento es un crecimiento tecnológico exógeno que puede ser resultado del contacto con otras economías, como es el caso de países menos desarrollados, y que puede tener implicaciones de políticas diferentes.

En este modelo discreto el comportamiento de cada tipo de familia puede reducirse a un sistema de dos variables.

En el segundo apartado presentamos el modelo a detalle. En el tercero, los resultados del cálculo numérico del mismo para casos de economía abierta y cerrada al flujo de capital físico, donde estudiamos también el efecto de la relajación de la restricción de crédito. Finalmente, en el cuarto apartado se discuten los resultados.

\section{EL MODELO}

\subsection{LA OPTIMIZACIÓN DE LAS FAMILIAS}

Consideramos un modelo de tres generaciones traslapadas para el que cada generación consiste de individuos idénticos que viven durante tres períodos. Expon- 
dremos los egresos e ingresos de la familia en cada generación como si fuera un sólo agente. Este planteamiento es equivalente al que hacen Abel $(1985,1987)$ y Kimball (1987) en el caso en que la utilidad aditiva, los viejos y los adultos tienen el mismo interés los unos por los otros.

En el período $t$ la familia consiste de infantes, adultos y viejos. Los egresos de la familia son: $c_{0 t}$, el consumo de los infantes y $h_{t}$, el monto de inversión en servicios educativos; con $c_{1 t}$ y $c_{2 t}$ denotamos el consumo de los adultos y de los viejos, respectivamente; $\mathrm{y}$, con $s_{t}$ el ahorro. Cabe señalar que cada una de estas variables está considerada en términos per cápita. Los ingresos de la familia son un monto $\Gamma_{t} \cdot s_{t-1}$, donde

$$
\Gamma_{t}=\frac{1+r_{K t}}{1+n}
$$

Esta expresión representa, corregidos a términos per cápita, los intereses percibidos por los ahorros de la familia en la generación anterior; $r_{H t} \cdot H_{t}$, es el rendimiento del capital humano efectivo que poseen los adultos, donde $H_{t}=H\left(h_{t-1}\right)$ es el capital humano efectivo, resultado del aprovechamiento de los servicios educativos por los adultos cuando eran infantes.

Respecto de la función $H$ de aprovechamiento del servicio educativo (o función de producción de capital humano en base al insumo), suponemos que satisface las condiciones de Inada. Una implicación de esto es que $H^{\prime}\left(h_{t-1}\right)$ tiende a infinito si $h_{t-1}$ tiende a cero. Puesto que esto es una hipótesis irreal en cuanto a la inversión en la educación formal, podemos suponer que la inversión en capital humano solamente representa educación formal si $h_{t-1} \geq h_{f}$, donde $h_{f}$ representa el nivel de gasto en capital humano mínimo para involucrar educación formal.

Por otro lado, la restricción de presupuesto de la familia en el tiempo $t$ es:

$$
(1+n) c_{0 t}+c_{1 t}+\frac{1}{1+n} c_{2 t}+s_{t}+(1+n) h_{t}=\Gamma_{t} \cdot s_{t-1}+r_{H t} \cdot H_{t}
$$

Como referencia definimos la transferencia que hacen los viejos a los jóvenes en la generación $t$ :

$$
b_{t}=\Gamma_{t} \cdot s_{t-1}-\frac{1}{1+n} c_{2 t}
$$


Por último, las familias enfrentan la restricción al crédito

$$
s_{t} \geq s_{0 t^{\prime}}
$$

donde $s_{0 t} \leq 0$.

Consideramos que en el período $t$ la familia maximiza la siguiente función de utilidad:

$$
U_{t}=\sum_{i=0}^{\infty} \mu^{i} V_{t+i^{\prime}}
$$

donde

$$
V_{t}=V\left(c_{0 t}, c_{1 t}, c_{2 t}\right)
$$

Suponemos que $V_{t}$ tiene las propiedades usuales, es decir, es creciente en cada variable y es cóncava. Asimismo se demuestra que las condiciones de primer orden del modelo son las siguientes:

$$
\begin{gathered}
(1+n) V_{t, 1}=V_{t, 0} \\
(1+n) V_{t, 2}=V_{t, 1} \\
V_{t, 1}=\mu \Gamma_{t} V_{t+1,1}+\eta_{s t} \\
(1+n) V_{t, 1}=\mu r_{H t+1} H_{t+1}^{\prime} V_{t+1,1}
\end{gathered}
$$

donde $\eta_{s t}$ es el multiplicador de Lagrange asociado con la restricción del ahorro.

Las condiciones (1.7) y (1.8) establecen la relación entre la utilidad marginal de los adultos y la de sus hijos y los viejos. La condición (1.9) es equivalente a la que obtiene Diamond (1965) pero no resuelve el problema debido a la presencia del multiplicador $\eta_{s t}$. La condición (1.10) establece la tasa de crecimiento óptima 
de la utilidad entre generaciones a través de una tasa de decrecimiento de la utilidad marginal del consumo en el período 1, en términos del rendimiento del capital humano en lugar de la tasa de interés debido a que el primero es mayor.

Comparando las ecuaciones (1.9) y (1.10) obtenemos

$$
\begin{aligned}
& \eta_{s t}=0 \Leftrightarrow r_{H t+1} H_{t+1}^{\prime}=1+r_{K t+1} \\
& \eta_{s t}>0 \Leftrightarrow r_{H t+1} H_{t+1}^{\prime}>1+r_{K t+1}
\end{aligned}
$$

Es decir, mientras la capacidad económica de la familia no llegue al nivel en que su inversión en capital humano reduzca el rendimiento marginal al de la tasa de interés del ahorro monetario, no ahorra $\mathrm{y}$, por el contrario pide prestado si $s_{0 t}<0$; y, cuando su riqueza cubre sus necesidades de consumo, ahorra igualando el rendimiento de ambos tipos de inversión.

Para simplificar el análisis del modelo utilizaremos la función de utilidad separable

$$
V_{t}=u\left(c_{0 t}\right)+u\left(c_{1 t}\right)+u\left(c_{2 t+1}\right)
$$

donde

$$
u(c)=\frac{c^{1-\sigma}-1}{1-\sigma}
$$

Las ecuaciones (1.7), (1.8) y (1.10) dan lugar al siguiente sistema de consumo:

$$
\begin{aligned}
& \frac{c_{0 t}}{c_{1 t}}=(1+n)^{-1 / \sigma} \\
& \frac{c_{1 t}}{c_{2 t}}=(1+n)^{-1 / \sigma}
\end{aligned}
$$




$$
\frac{c_{1 t+1}}{c_{1 t}}=\left(\frac{\mu}{1+n} \cdot r_{H t+1} H_{t+1}^{\prime}\right)^{-1 / \sigma}
$$

\subsection{LA ECONOMÍA AGREGADA}

Supondremos que la economía se compone de familias con la misma función de utilidad divididas en las poblaciones 1 y 2 de acuerdo a sus recursos iniciales. Cada población es de tamaño $N_{t}^{1}$ y $N_{t}^{2}$ respectivamente; los superíndices representan la población a la que se esté haciendo referencia. Las familias de cada población tienen una cantidad uniforme de recursos iniciales. En la primera población los adultos heredan en el primer período recursos $b_{1}^{1}$, además del resultado de una inversión en capital humano que incluyen los títulos de propiedad sobre el capital inicial de la economía, $K_{1}$.

Definimos el capital humano agregado como la suma de los capitales humanos individuales; es así que

$$
H_{t}^{a}=N_{t}^{1} H\left(h_{t-1}^{1}\right)+N_{t}^{2} H\left(h_{t-1}^{2}\right)
$$

La función de producción agregada es

$$
Y_{t}=F\left(K_{t}, H_{T}^{a}\right)-\delta K_{t}
$$

$F$ es homogénea de grado 1 porque representa el agregado de las funciones de producción de los productores individuales bajo condiciones de competencia perfecta, y $\delta$ es la depreciación. El interés en una función general incluye investigar los efectos de la elasticidad de substitución entre $K_{t}$ y $H_{t}^{a}$. Sea

$$
\begin{gathered}
f(k)=F(k, 1) \\
g\left(k^{-1}\right)=F\left(1, k^{-1}\right)
\end{gathered}
$$


Pueden verificarse $g^{\prime}\left(k^{-1}\right)=f(k)-k \cdot f^{\prime}(k), f^{\prime \prime}, g^{\prime \prime}<0$. Ambas funciones, $f^{\prime}$ y $g^{\prime}$, son invertibles. Escribiendo $k_{t}=\frac{k_{t}}{H_{t}^{a}}$, la tasa de interés y el rendimiento del capital humano, determinados por las optimizaciones de los productores individuales, son:

$$
\begin{gathered}
r_{K t}=F_{K}\left(K_{t}, H_{t}^{a}\right)=f^{\prime}\left(k_{t}\right) \\
r_{H t}=F_{H}\left(K_{t}, H_{t}^{a}\right)=g^{\prime}\left(k_{t}^{-1}\right)
\end{gathered}
$$

Por lo tanto

$$
r_{H t}=R_{H K}\left(r_{K t}\right)=_{d e f} g^{\prime}\left(\frac{1}{f^{\prime-1}\left(r_{K t}\right)}\right)
$$

La función $R_{H K}$ definida aquí tiene derivada negativa: entre más alta es la tasa de interés, más bajo es el rendimiento del capital humano y viceversa.

Debido a que suponemos que $H\left(h_{t-1}\right)$ satisface las condiciones de Inada, nos limitaremos a

$$
H\left(h_{t-1}\right)=B \cdot e^{x t}\left(h_{t-1}^{a}\right)^{\chi} h_{t-1}^{\beta}
$$

tomando en cuenta uno de los dos casos

$$
\chi+\beta=1, \quad x=0 \quad \text { ó } \quad \chi+\beta<1, \quad x \geq 0
$$

donde

$$
h_{t}^{a}=\frac{H_{t}^{a}}{\left(N_{t}^{1}+N_{t}^{2}\right)}
$$


En el primer caso, $H$ induce un modelo de crecimiento endógeno, mientras que el segundo se refiere al crecimiento exógeno que puede aplicarse al caso en que las externalidades del conocimiento provengan de otras economías. Al considerar que la externalidad del conocimiento depende del nivel $h_{t}^{a}$ de capital humano per cápita de la economía, evitamos que la tasa de crecimiento dependa de la población de la economía. De cualquier forma, cuando las externalidades son consideradas en el nivel total de capital humano per cápita este efecto usualmente se atribuye a un conjunto de economías más que a una sola por lo que, este tipo de consideración podría incorporarse en la constante $B$.

Definimos

$$
\begin{gathered}
K_{t}=N_{t-1}^{1} \cdot s_{t-1}^{1}+N_{t-1}^{2} \cdot s_{t-1}^{2} \\
N_{t}=N_{t}^{1}+N_{t}^{2}
\end{gathered}
$$

además

$$
N_{t}^{1}=(1+n)^{t} \cdot N_{0}^{1}, \quad N_{t}^{2}=(1+n)^{t} \cdot N_{0}^{2}
$$

Consideraremos los casos de economías abiertas, cerradas y semiabiertas en que, arriba de cierta tasa de interés, ingresa el capital extranjero. Sea $K_{t}^{E}$ la cantidad neta de capital invertido desde el extranjero que será cero en el caso cerrado.

Como el producto neto es igual al ingreso, entonces

$$
F\left(K_{t}, H_{t}^{a}\right)=\sum_{i=1}^{2} N_{t}^{i}\left(r_{H t} \cdot H\left(h_{t-1}^{i}\right)+\frac{r_{K t}}{1+n} \cdot s_{t-1}^{i}\right)+r_{K t} K_{t}^{E}
$$

Puesto que no todas las familias ahorran, la variable $s_{t}$ no funciona como variable de estado por lo que, en su lugar, utilizamos la transferencia intergeneracional $b_{t}$. Sustituyendo la ecuación (1.3) en (1.2) podemos despejar el ahorro en la forma

$$
s_{t}^{i}=b_{t}^{i}+r_{H t} \cdot H\left(h_{t-1}^{i}\right)-(1+n) h_{t}^{i}-(1+n) c_{0 t}^{i}-c_{1 t}^{i}
$$


Sustituyendo esta expresión en la ecuación (1.3) obtenemos

$$
b_{t+1}^{i}=-\frac{1}{1+n} \cdot c_{2 t+1}^{i}+\Gamma_{t+1} \cdot s_{t}^{i}
$$

quedando una ecuación de transición de la variable de estado $b_{t}^{i}$.

\section{EL COMPORTAMIENTO DE LAS FAMILIAS CUANDO AHORRAN}

Las familias de cualquier población toman sus decisiones en base a las trayectorias conocidas de $r_{K}$ y $r_{H}$. Analizamos el caso de una familia que ahorra, es decir, que decide $s_{t}>s_{0 t}$ a partir del período $\tau$. En este caso $c_{0}, c_{1}, c_{2}$, de manera balanceada, se multiplican en cada período por $\gamma_{t+1}$, donde utilizando las ecuaciones (1.11) a (1.16) queda:

$$
\gamma_{t+1}=\left(\mu \Gamma_{t+1}\right)^{1 / \sigma}
$$

A partir de la condición de eficiencia (1.16) se obtiene la inversión óptima en capital humano,

$$
h_{t-1}=\left(\beta B e^{x t}\left(H_{t-1}^{a}\right)^{\chi} \frac{r_{H t}}{1+r_{K t}}\right)^{1 /(1-\beta)}
$$

La cantidad óptima de capital humano per cápita es independiente de la riqueza de la familia. A partir de las ecuaciones (3.2) y (2.5) ó (2.6), $h_{t-1}$ puede expresarse como función de $r_{K t}$ ó $k_{t}$.

Por otro lado podemos reducir el problema del ahorrador, y posteriormente también el del no ahorrador, a uno de dos variables, $b_{t}$ y $c_{2 t}$. De las condiciones de primer orden (1.19) y (1.21) obtenemos la siguiente identidad

$$
(1+n) c_{0 t}+c_{1 t}=\beta_{1}\left(1+r_{K t+1}\right)^{-1 / \sigma} c_{2 t+1}
$$


donde

$$
\beta_{1}=\left((1+n)^{-(1-\sigma) / \sigma}+1\right) \mu^{-1 / \sigma}
$$

$\mathrm{y}$

$$
r_{H t} H_{t}=r_{H t} H^{\prime}\left(h_{t-1}\right) \beta^{-1} h_{t-1}=\beta^{-1}\left(1+r_{K t}\right) h_{t-1}
$$

y simplificando queda

$$
a_{t+1}=\frac{1}{1+n}\left(1+\beta_{1}\left(1+r_{K t+1}\right)^{-(1-\sigma) / \sigma}\right)
$$

De las ecuaciones (2.15), (2.16) y (1.1) se obtiene

$$
b_{t+1}=\Gamma_{t+1} b_{t}-a_{t+1} c_{2 t+1}+(1+n) \Gamma_{t+1}\left(\beta^{-1} \Gamma_{t} h_{t-1}-h_{t}\right)
$$

En los casos en que el modelo es de crecimiento encontraremos, consecutivamente, una tasa de crecimiento de equilibrio. Hacemos el cambio de variables en (3.7),

$$
c_{i t}=\bar{c}_{i t} \theta^{t}, \quad i=0,1,2 \quad s_{t}=\bar{s}_{t} \theta^{t} \quad h_{t}=\bar{h}_{t} \theta^{t} \quad h_{t}=\bar{h}_{t} \theta^{t}
$$

de donde obtenemos

$$
\overline{\Gamma_{t}}=\Gamma_{t} \theta^{-1}
$$

Posteriormente tomaremos $\theta$ como la tasa de crecimiento de equilibrio. La ecuación (3.7) toma la forma

$$
b_{t+1}=\Gamma_{t+1} \bar{b}_{t}-a_{t+1} \bar{c}_{2 t+1}+(1+n) \Gamma_{t+1}\left(\beta^{-1} \Gamma_{t} \bar{h}_{t-1}-\bar{h}_{t}\right)
$$

Resolvemos a continuación esta ecuación en diferencias. Sea

$$
\Pi_{0}^{\Gamma}=1 \quad \Pi_{t}^{\Gamma}=\left(\Pi_{i=1}^{t} \bar{\Gamma}_{i}\right)^{-1}
$$


Para $t \geq \tau$, multiplicando por $\Pi_{t+1}^{\Gamma}$,

$$
\Pi_{t+1}^{\Gamma} \bar{b}_{t+1}=\Pi_{t}^{\Gamma} \bar{b}_{t}-\Pi_{t+1}^{\Gamma} a_{t} \bar{c}_{2 t+1}+(1+n)\left(\beta^{-1} \Pi_{t-1}^{\Gamma} \bar{h}_{t-1}-\Pi_{t}^{\Gamma} \bar{h}_{t}\right)
$$

Sumando desde $\tau$ hasta infinito, cancelando y reacomodando términos tenemos

$$
\sum_{t=\tau+1}^{\infty} \Pi_{t}^{\Gamma} a_{t} \bar{c}_{2 t}=\Pi_{\tau}^{\Gamma} \bar{b}_{\tau}+(1+n) \beta^{-1} \Pi_{\tau-1}^{\Gamma} \bar{h}_{t-1}+(1+n)\left(\beta^{-1}-1\right) \sum_{t=\tau}^{\infty} \Pi_{t}^{\Gamma} \bar{h}_{t}
$$

ó, escribiendo

$$
\Pi_{0}^{\gamma}=1, \quad \Pi_{t}^{\gamma}=\Pi_{i=1}^{t} \bar{\gamma}_{i}
$$

Tomando en cuenta el sistema (1.14) a (1.16) y las ecuaciones (3.1) y (3.8), obtenemos la siguiente aproximación para la trayectoria del consumo cuya tasa viene dada por

$$
\begin{gathered}
\bar{c}_{2 t+1}=\left(\Pi_{t+1}^{\gamma} / \Pi_{\tau+1}^{\gamma}\right) \bar{c}_{2 \tau+1}, \quad t \geq \tau \\
\left(\bar{c}_{2 \tau+1} / \Pi_{\tau+1}^{\gamma}\right) \sum_{t=\tau+1}^{\infty} \Pi_{t}^{\Gamma} a_{t} \Pi_{t}^{\gamma}=\Pi_{\tau}^{\Gamma} \bar{b}_{\tau}+(1+n) \beta^{-1} \Pi_{\tau-1}^{\Gamma} \bar{h}_{t-1}+(1+n)\left(\beta^{-1}-1\right) \sum_{t=\tau}^{\infty} \Pi_{t}^{\Gamma} \bar{h}_{t}
\end{gathered}
$$

Esta ecuación es análoga a la restricción de riqueza de los modelos de crecimiento endógeno, es decir, a una condición de No Ponzi. Expresa que el valor presente del consumo per cápita de los niños, adultos y viejos de una familia es igual a la riqueza de la dinastía, que consiste de la suma de su herencia inicial y de sus ingresos netos provenientes de la inversión en capital humano. La familia escoge el nivel general de consumo, representado en este caso por $\bar{c}_{2 t+1}^{i}$, igualando gastos e ingresos.

\section{EL COMPORTAMIENTO DE LAS FAMILIAS CUANDO NO AHORRAN}

Estudiamos la ecuación de la variable de estado $b_{t}$ también en el caso en que una familia no ahorra, es decir, cuando decide que $s_{t}=s_{0 t}$. Extendemos el cambio de variables (3.8) a $s_{0 t}=\bar{s}_{0 t} \theta^{t}$. Entonces la ecuación (2.16) es: 


$$
\bar{b}_{t+1}=-\frac{1}{1-n} \bar{c}_{2 t+1}+\bar{\Gamma}_{t+1} \bar{s}_{0 t}
$$

Aplicando las ecuaciones (1.14) y (1.25), obtenemos

$$
(1+n) c_{0 t}+c_{1 t}+\frac{1}{1+n} c_{2 t}=\beta_{2} c_{2 t}
$$

donde

$$
\beta_{2}=(1+n)^{-(2-\sigma) / \sigma}+(1+n)^{-1 / \sigma}+(1+n)^{-1}
$$

Escribiendo $\bar{H}\left(h_{t-1}\right)=H\left(h_{t-1}\right) \theta^{t}$, la ecuación (2.15) toma la forma:

$$
(1+n) \bar{h}_{t}=r_{H t} \bar{H}\left(h_{t-1}\right)-\beta_{2} \bar{c}_{2 t}+\left(\bar{\Gamma}_{t} \bar{s}_{0 t-1}-\bar{s}_{0 t}\right)
$$

Para $c_{2 t}$ obtenemos de (1.15), (1.16) y (3.1) la ecuación de transición

$$
\bar{c}_{2 t+1}=\bar{\gamma}_{t+1} \bar{c}_{2 t}
$$

Las ecuaciones (4.1), (4.3) y (4.4) resuelven iterativamente $h_{t}, b_{2 t+1}$ y $c_{2 t+1}$, hasta el momento en que $r_{H t} H^{\prime}\left(h_{t-1}\right)<1+r_{K t}$, en cuyo caso la familia se convierte en una familia ahorradora.

\section{LAS TRAYECTORIAS DE CRECIMIENTO BALANCEADO}

En un estado estacionario todas las familias son ahorradoras aunque su ahorro sea justamente $s_{0 t}$. Esto se debe a que la tasa de crecimiento del consumo de ambas poblaciones debe ser igual y, por lo tanto, $\eta_{s t}=0$ (ecuaciones (1.1) y (1.14) a (1.16)). En un estado estacionario el nivel óptimo de la inversión en capital humano no depende de la riqueza (ecuación (3.2)) pero sí de la cantidad agregada $H_{t}^{a}$. De las ecuaciones (2.8), (2.10) y (3.2) obtenemos 


$$
h_{t}^{a}=\left(B e^{x t}\right)^{1 /(1-\beta)}\left(\frac{\beta r_{H t}}{1+r_{K t}}\right)^{\beta /(1-\beta)} \cdot\left(h_{t-1}^{a}\right)^{\chi /(1-\beta)}
$$

Suprimiendo el índice $t$ para las cantidades estacionarias, en el caso en que $\chi+\beta=1$ y $x=0$, sucederá

$$
\gamma=B^{1 /(1-\beta)}\left(\frac{\beta r_{H t}}{1+r_{K t}}\right)^{\beta /(1-\beta)}
$$

En el caso $\chi+\beta<1$ y $\quad x \geq 0$, dividiendo la ecuación (5.1) en $t+1$ por la misma ecuación en $t \mathrm{y}$ simplificando, tendremos que

$$
\gamma=e^{x /(1-\beta-\chi)}
$$

Como además tenemos la ecuación (3.1), la tasa de interés de equilibrio satisface, en el caso $\chi+\beta=1$ y $x=0$,

$$
1+r_{K}=\left(\frac{1+n}{\mu}\right) \cdot e^{\sigma x /(1-\beta-\chi)}
$$

Tenemos del lado izquierdo una función decreciente y del derecho una creciente de la razón capital-capital humano (ecuaciones (2.4) y (2.5)), por lo que existe una única solución $k$, y por lo tanto $r_{K} \mathrm{y} r_{H}$.

En el caso $\chi+\beta<1$ y $x \geq 0$, obtenemos análogamente

$$
\left(1+r_{K}\right)^{1-\beta+\sigma \beta}=\left(\frac{1+n}{\mu}\right)^{1-\beta} \cdot B^{\sigma}\left(\beta r_{H t}\right)^{\beta \sigma}
$$

que también tiene una única solución $k$, y por lo tanto $r_{K} \mathrm{y} r_{H}$.

Escribamos ahora $\theta=\gamma$. Entonces de la (3.1) y (3.8) conseguimos

$$
\bar{\Gamma}=\gamma^{-(1-\sigma)} \mu^{-1}
$$


Suponemos que $\gamma^{(1-\sigma)} \mu<1$ para que sea convergente la utilidad. Por lo tanto $\bar{\Gamma}>1$. Lo anterior implica que,

$$
\Gamma>(1+n)^{-1} \Rightarrow \Gamma>\gamma(1+n)^{-1} \Rightarrow 1+r_{K}>\gamma
$$

También, es conveniente simplificar la definición (3.6) en

$$
a=(1+n)^{-1}+\beta_{3} \gamma^{-1}
$$

donde

$$
\beta_{3}=(1+n)^{-(2-\sigma) / \sigma}+(1+n)^{-1 / \sigma}
$$

por lo que $\beta_{2}=\beta_{3}+(1+n)^{-1}, \mathrm{y}$

$$
\beta_{1}\left(1+r_{K}\right)^{-1 / \sigma}=\beta_{3} \gamma^{-1}
$$

A partir de la ecuación (3.15), recordando que se trata de un estado estacionario, tenemos

$$
\bar{c}_{2} a \Pi_{\tau}^{\Gamma}(\bar{\Gamma}-1)^{-1}=\Pi_{\tau}^{\Gamma} \bar{b}+(1+n) \beta^{-1} \Pi_{\tau-1}^{\Gamma} \bar{h}+(1+n)\left(\beta^{-1}-1\right) \bar{h} \Pi_{\tau}^{\Gamma}\left(1-\bar{\Gamma}^{-1}\right)^{-1}
$$

Dividiendo entre $\Pi_{\tau}^{\Gamma}$ y simplificando, queda

$$
a \bar{c}_{2}-(\bar{\Gamma}-1) \bar{b}=(1+n) \bar{h} \bar{\Gamma}\left(\beta^{-1} \bar{\Gamma}-1\right)
$$

Los diferentes pares de soluciones $(\bar{c}, \bar{b})$ corresponden a diferentes niveles de riqueza de la población. Por otra parte, sustituyendo las ecuaciones (3.3) y (3.5) en la (2.15), y utilizando (5.8), obtenemos

$$
\beta_{3} \gamma^{-1} \bar{c}_{2}-\bar{b}=(1+n)\left(\beta^{-1} \bar{\Gamma}-1\right) \bar{h}-\bar{s}
$$

Resolvemos las ecuaciones simultáneas (5.9) y (5.10), donde el determinante es 


$$
\left.\Delta=\beta_{3} \gamma^{-1}(\Gamma-1)-a=\beta_{3} \gamma^{-1}(\Gamma-1)-(1+n)^{-1}-\beta_{3} \Gamma\right)=\beta_{3}\left(\gamma^{-1}-1\right)(\Gamma-1)-(1+n)^{-1}-\beta
$$

Puesto que $\bar{\Gamma}>1$, para el caso de crecimiento $\gamma \geq 1$ el determinante es negativo. Las soluciones de (5.9) y (5.10) son

$$
\begin{gathered}
\bar{c}_{2}=-\Delta^{-1}\left((1+n)\left(\beta^{-1} \bar{\Gamma}-1\right) \bar{h}+(\bar{\Gamma}-1) \bar{s}\right) \\
\bar{b}=-\Delta^{-1}\left\{\left((1+n) \beta_{3}\left(\gamma^{-1}-1\right) \bar{\Gamma}-1\right)\left(\beta^{-1} \bar{\Gamma}-1\right) \bar{h}+a \bar{s}\right\}
\end{gathered}
$$

El caso $\Delta<0$, no tiene sentido si las familias con capital físico y humano positivos consumen positivamente. Obsérvese que $c_{2}$ es positivo aún si el ahorro es cero, y que la herencia cambia, en relación al capital humano, de negativa a positiva con el ahorro. Hay que recordar que $h$ depende de los parámetros a través de la solución $k \mathrm{y}$, por lo tanto, depende no solamente de los parámetros individuales sino también del promedio del capital per cápita de la economía, de donde tenemos que

$$
\begin{gathered}
k \frac{K_{t}}{H_{t}^{a}}=\frac{N_{t-1}^{1} s_{t-1}^{1}+N_{t-1}^{2} s_{t-1}^{2}}{N_{t}^{1} H_{t}^{1}+N_{t}^{2} H_{t}^{2}}=\frac{\left(N_{0}^{1} s^{-1}+N_{0}^{2} s^{-2}\right) \gamma^{t-1}}{(1+n)\left(N_{0}^{1}+N_{0}^{2}\right) H_{t}} \\
\bar{h}=\frac{\beta}{(1+n) k} \cdot \frac{r_{H}}{1+r_{k}} \cdot \frac{N_{0}^{1} s^{-1}+N_{0}^{2} s^{-2}}{N_{0}^{1}+N_{0}^{2}}
\end{gathered}
$$

Las ecuaciones (5.14) y (5.15) se obtuvieron mediante el uso del software $\mathrm{Ma}$ thematic versión $4.0 \mathrm{y}$ describen los niveles óptimos de acervo de capital físico y humano, respectivamente.

\section{CONCLUSIONES}

El modelo reproduce la idea intuitiva de que una familia de pocos recursos puede decidir dedicar su inversión al capital humano y por lo tanto no ahorrar. Esto su- 
cede debido a la restricción de crédito, pero incluso puede darse el caso de cero ahorro en equilibrio con la restricción en el punto de no ser efectiva en estado de igualdad.

Se analizó primero la economía cerrada al flujo de capital físico. Cuando la economía sufre un impacto positivo a su productividad sube la tasa de interés. La distribución de riqueza afecta la tasa de interés, subiendo más si es más igualitaria debido a que la población más rica está más dispuesta al ahorro. Si la restricción de crédito es relajada suben más los intereses debido al incremento en la demanda de crédito, pero esta vez el impacto puede revertir ligeramente el efecto de la distribución. Durante este período se puede dar una diferenciación en la cantidad de capital humano que tiene la población rica comparada con la pobre, que es mayor entre mayor sea la desigualdad de la distribución y que tiende a revertirse si se relaja la restricción de crédito. Esto se atribuye a que la población rica puede realizar el nivel de inversión óptimo, mientras que la pobre debe acumularlo a través de varios períodos. Mientras esto sucede el capital humano es más escaso y la población rica invierte relativamente más, compensando el déficit de capital humano de la población pobre.

Al relajarse la restricción de crédito se forma menos capital físico en la economía cerrada. En cambio en la economía abierta se forma más capital humano de manera más rápida, aunque luego se produce menos porque persiste el aumento en la tasa de interés. La asignación de recursos es más eficiente pues, la población pobre puede invertir más en capital humano con un rendimiento mayor. Sin embargo, aunque esta población adelanta su consumo hacia el presente durante el período en que se forma su capital humano y no ahorra, su utilidad disminuye mientras que la de la población rica se incrementa. Este efecto paradójico, en el que cuando existe la restricción cada familia de la población 2 quisiera el crédito pero si se relaja la restricción la población en su conjunto pierde utilidad, se debe a que la restricción de crédito hace escaso el capital humano y el uso de la porción de su función de producción que tiene mayores rendimientos y, por lo tanto, lo sube de valor. En los casos en que ésta hace que tiendan a disminuir los ingresos de la población pobre, una política que relaje la restricción de crédito, que podría consistir, por ejemplo, de gasto gubernamental en la educación financiado por medio de préstamos pa- 
gados en períodos posteriores, si bien incrementa la eficiencia de la economía, solamente resulta en un incremento de Pareto en el bienestar si es acompañada de una política redistributiva de los costos, consistente en que la población rica pague una mayor proporción de estos.

Así pues, el comportamiento de la economía abierta al flujo de capital físico debe ser suave, mostrando que el comportamiento caótico tiene que ver con el mecanismo de precios y no con el de decisión individual. En la economía abierta la población 2 no ahorra y, dependiendo del nivel de riqueza ésta se aproxima al nivel óptimo de capital humano más rápidamente.

Al comparar el desarrollo de la economía abierta o cerrada entran en juego efectos distributivos. Como en la economía cerrada sube la tasa de interés, el valor presente de una unidad de capital es mayor en la economía cerrada que en la abierta. Ésta se incrementa aún más en caso de relajarse la restricción de crédito.

Subrayemos los resultados principales. Como ya hemos dicho, una familia de pocos recursos dedica su inversión al capital humano y por lo tanto no ahorra. Esto sucede aún más en una economía abierta en que la tasa de interés del exterior es más baja que la doméstica. En este caso el sector pobre transita toda la trayectoria hasta el equilibrio sin ahorrar.

En la economía cerrada observamos que el capital físico crece menos cuando se relaja la restricción de crédito. De Gregorio (1996), por ejemplo, encuentra que la restricción de crédito tiene efectos ambiguos sobre el crecimiento. Sin embargo, al incluir el estudio de la distribución encontramos que la preferencia de los agentes sobre la restricción de crédito, así como el efecto sobre su ahorro, depende de su riqueza. Puede darse el resultado paradójico en el cual, si se relaja la restricción de crédito el aumento en eficiencia puede no resultar en un aumento de Pareto del beneficio, perjudicando a la población pobre si no se toman medidas redistributivas en la repercusión del pago de los créditos. Esto resulta de la restricción de crédito que escasea el capital humano. Al relajarse, el efecto sobre la riqueza de la población pobre depende de la elasticidad de los rendimientos del capital humano. Por otra parte, al relajar la restricción el consumo se trae hacia el presente, lo cual significa una mayor tasa de crecimiento en el presente y una menor en el futuro. 
La economía abierta converge más rápidamente que la cerrada y el sector más pobre de la población obtiene mayor utilidad en ella, mientras que los sectores ricos pierden los beneficios de las altas tasas de interés. Un costo de la política de apertura es que una buena proporción del capital físico sea propiedad del exterior. Otro aspecto que cabe mencionar sobre el caso de economía abierta es que, no hemos tomado en cuenta el problema de transferencia del capital del exterior, tanto en sus costos como en sus posibles repercusiones de tipo comercial. En la economía abierta la población pobre dedica su inversión al capital humano. En la cerrada, pasa más rápidamente al estado del ahorro pero a niveles de acumulación más bajos.

Una consecuencia interesante del modelo es que los sectores pobres de la economía, para quienes resulta óptimo gastar todo su ingreso en rubros que incluyen la inversión en capital humano, tienen por lo mismo una alta propensión al consumo ante las fluctuaciones económicas (en este modelo estrictamente es 1) por lo que, su comportamiento se aproxima al modelo Keynesiano de consumo mientras que los sectores ricos, quienes tienen incentivos para ahorrar, suavizan su consumo y por lo tanto tienden al concepto de consumo permanente de Friedman. Consecuentemente, ante las fluctuaciones, una función agregada de consumo sería una ponderación de estas dos, de acuerdo al tamaño y riqueza de las poblaciones. Esto significaría que, al ir creciendo y desarrollándose una economía el comportamiento agregado del consumo tendería a pasar del primer al segundo modelo.

Posiblemente el comportamiento caótico desaparecería si los sectores de población no consistieran de agentes idénticos que actúan de manera sincronizada y en bloque. Sin embargo, no dejan de existir situaciones en que sectores sujetos a situaciones económicas comunes, de cualquier índole, actúan en bloque y por lo tanto podrían generar algún tipo de caos por algún tiempo. Asimismo, es interesante que este tipo de fluctuaciones puedan ser de mayor amplitud cuando una mayor parte de los agentes económicos actúan sujetos a ciertas restricciones, como las resultantes de la pobreza, logrando que la elasticidad de las tasas de interés sea mayor. Este tipo de lógica podría explicar la mayor intensidad de los ciclos económicos que sufrían en siglos pasados los países ahora desarrollados. 
FORMACIÓN DE CAPITAL HUMANO Y AHORRO INTERGENERACIONAL

\section{BIBLIOGRAFÍA}

Abel D. y R. Kimball (1987), "The Economic Implications of Learning by Doing”, Review of Economic Studies 29, 1962, pp. 155-173.

Aghion, P. y P. Howitt (1992), "A model of growth through creative destruction", Econometrica 60, pp. 323-702.

Arnold, L. (1998), "Growth, welfare, and trade in an integrated model of humancapital accumulation and research", Journal of Macroeconomics 20, pp. 81105.

Barro, Robert J.; N. Gregory Mankiw y Xavier Sala-i-Martin (1992), “Capital Mobility in Neoclassical Models of Growth", National Bureau of Economic Research, Working Paper No. 4206.

Becker, G. (1971), The Economies of Discrimination. Chicago: Chicago University Press, 2nd ed.

Caballé, J. y M. S. Santos (1993), "On endogenous growth with physical and human capital", Journal of Political Economy 101, pp. 1042-1067.

Funke, M y H. Strulik (2000), "On endogenous growth with physical capital, human capital and product variety", European Eonomic Review 44, pp. 491515 .

Diamond, P. (1965), "National Debt in a neoclassical growth model", American Economic Review 41, 1126-50.

Griliches, Z. (1979), "Issues in Assessing the Contribution of Research and Development to Productivity Growth", Bell Journal of Economics 10, pp. 92-116.

Grossman, G.M. y E. Helpman (1991), Innovation and Growth in the Global Economy, Cambridge, MA: MIT Press.

Jappelli, Tullio y Marco Pagano (1994), "The Welfare Effects of Liquidity Constraints," mimeo.

Keller, W. (1996), "Absorptive capacity: On the creation and acquisition of technology in development", Journal of Development Economics 49, pp. 199227.

Lucas, R. E. Jr. (1988), "On the Mechanics of Development Planning”, Journal of Monetary Economics 22, pp. 3-42. 
Mayer D. Foulkes (1997), "Sectoral Public Power and Endogenous Growth", Economia Mexicana NUEVA EPOCA, vol. 0 (1), Enero-Julio, pp. 33-81.

Modigliani, F. (1985), "Measuring the Contribution of Intergenerational Transfers to Total Wealth: Conceptual Issues and Empirical Findings", paper presented at Modeling the Accumulation and Distribution of Personal Wealth seminar, Paris, France, September 10-11.

Rebelo, S. (1991), "Long-Run Policy Analysis and the Long-Run Growth", Journal of Political Economy 99, 3, pp. 500-521.

Romer, P. (1988), "Increasing Returns and Long-Run Growth", Journal of Political Economy, October, 1986.

Romer, P. (1990), "Endogenous technological change", Journal of Political Economy 98 , pp. 71-102.

Uzawa, H. (1965), "Optimal technical change in an aggregative model of economic growth”, International Economic Review 6, pp. 18-31. 